Pacific Journal of Mathematics

DEDEKIND DOMAINS AND RINGS OF QUOTIENTS 


\section{DEDEKIND DOMAINS AND RINGS OF QUOTIENTS}

\section{LUTHER CLABORN}

We study the relation of the ideal class group of a Dedekind domain $A$ to that of $A_{S}$, where $S$ is a multiplicatively closed subset of $A$. We construct examples of (a) a Dedekind domain with no principal prime ideal and (b) a Dedekind domain which is not the integral closure of a principal ideal domain. We also obtain some qualitative information on the number of non-principal prime ideals in an arbitrary Dedekind domain.

If $A$ is a Dedekind domain, $S$ the set of all monic polynomials and $T$ the set of all primitive polynomials of $A[X]$, then $A[X]_{S}$ and $A[X]_{T}$ are both Dedekind domains. We obtain the class groups of these new Dedekind domains in terms of that of $A$.

1. Lemma 1-1. If $A$ is a Dedekind domain and $S$ is a multiplicatively closed sot of $A$ sush that $A_{S}$ is not a field, then $A_{S}$ is also a Dedekind domain.

Proof. That $A_{S}$ is integrally closed and Noetherian if $A$ is, follows from the general theory of quotient ring formations. The primes of $A_{S}$ are of the type $P A_{S}$, where $P$ is a prime ideal of $A$ such that $P \cap S=\dot{\phi}$. Since height $P A_{S}=$ height $P$ if $P \cap S=\phi, P \neq(0)$ and $P \cap S=\dot{\phi}$ imply that height $P A_{S}=1$.

Propjsition 1-2. If $A$ is a Dedekind domain and $S$ is a multiplicatively closed set of $A$, the assignment $C \rightarrow C A_{S}$ is a mapping of the set of fractionary ideals of $A$ onto the set of fractionary ideals of $A_{S}$ which is a homomorphism for multiplication.

Proof. $C$ is a fractionary ideal of $A$ if and only if there is a $d \in A$ such that $d C \cong A$. If this is so, certainly $d C A_{S} \leqq A_{S}$, so $C A_{S}$ is a fractionary ideal of $A_{S}$. Clearly $(B \cdot C) A_{S}=B A_{S} \cdot C A_{S}$, so the assignment is a homomorphism. Let $D$ be any fractionary ideal of $A_{S}$. Since $A_{S}$ is a Dedekind domain, $D$ is in the free group generated by all prime ideals of $A_{S}$, i.e. $D=Q_{i}^{e_{1}} \cdots Q_{k}^{e_{k}}$. For each $i=1, \cdots, k$ there is a prime $P_{i}$ of $A$ such that $Q_{i}=P_{i} A_{S}$. Set $E=P_{1}^{e_{1}} \cdots P_{k}^{\circ}$. Then using the fact that we have a multiplicative homomorphism of fractionary ideals, we get

Received December 13, 1963. 


$$
E A_{S}=\left(P_{1} A_{S}\right)^{e_{1}} \cdots\left(P_{k} A_{S}\right)^{e_{k}}=Q_{1}^{e_{1}} \cdots Q_{k}^{e_{k}} .
$$

CoRollary 1-3. Let $A$ be a Dedekind domain and $S$ be a multiplicatively closed set of $A$. Let $\bar{C}$ (for $C$ a fractionary ideal of $A$ or $A_{S}$ ) denote the class of the ideal class group to which $C$ belongs. Then the assignment $\bar{C} \rightarrow \overline{C A}_{S}$ is a homomorphism $\varphi$ of the ideal class group of $A$ onto that of $A_{S}$.

Proof. It is only necessary to note that if $C=d A$, then $C A_{S}=$ $d A_{S}$.

THEOREM 1-4. The kernel of $\phi$ is generated by all $\bar{P}_{\alpha}$, where $P_{\alpha}$ ranges over all primes such that $P_{\alpha} \cap S \neq \phi$.

If $P_{\alpha} \cap S \neq \phi$, then $P_{a} A_{S}=A_{S}$. Suppose $C$ is a fractionary ideal such that $\bar{C}=\bar{P}_{a}$, i.e. $C=d P_{\alpha}$ for some $d$ in the quotient field of $A$. Then $C A_{S}=d P_{a} A_{S}=d A_{S}$, and thus $\bar{C} A_{S}$ is the principal class.

On the other hand, suppose that $C$ is a fractionary ideal of $A$ such that $C A_{S}=x A_{S}$. We may choose $x$ in $C$. Then $C^{-1} \cdot x A$ is an integral ideal of $A$, and $\left(C^{-1} \cdot x A\right) A_{S}=A_{S}$. In other words, $C^{-1} \cdot x A=$ $P_{1}^{f_{1}} \cdots P_{l}^{f_{l}}$, where $P_{i} \cap S \neq \phi, i=1, \cdots, l$. Then $\bar{C}=\bar{P}_{1}^{-f_{1}}, \cdots,-\bar{P}_{l}^{-f_{l}}$, completing the proof.

ExAMPLE 1-5. There are Dedekind domains with no prime ideals in the principal class.

Let $A$ be any Dedekind domain which is not a principal ideal domain. Let $S$ be the multiplicative set generated by all $\Pi_{\alpha}$, where $\Pi_{\alpha}$ ranges over all the prime elements of $A$. Then by Theorem 1-4, $A_{S}$ will have the same class group as $A$ but will have no principal prime ideals.

Corollary 1-6. If $A$ is a Dedekind domain which is not a principal ideal domain, then $A$ has an infinite number of nonprincipal prime ideals.

Proof. Choose $S$ as in Example 1-5. Then $A_{S}$ is not a principal ideal domain, hence has an infinite number of prime ideals, none of which are principal. These are of the form $P A_{s}$, where $P$ is a (nonprincipal) prime of $A$.

Corollary 1-7. Let $A$ be a Dedekind domain with torsion class group and let $\left\{P_{a}\right\}$ be a collection of primes such that the subgroup of the ideal class group of $A$ generated by $\left\{\bar{P}_{\alpha}\right\}$ is not the entire 
class group. Then there are always an infinite number of nonprincipal primes not in the set $\left\{P_{\alpha}\right\}$.

Proof. For each $\alpha$, chose $n_{\alpha}$ such that $P_{\alpha}^{n_{\alpha}}$ is principal, say $=$ $A \cdot a_{\alpha}$. Let $S$ be the multiplicatively closed set generated by all $a_{\alpha}$. By Theorem 1-4, $A_{S}$ is not a principal ideal domain, hence $A_{S}$ must have an infinite number of non-principal prime ideals by Corollary 1-6. These come from non-principal prime ideals of $A$ which do not meet $S$. Each $P_{\alpha}$ does meet $S$, so there are an infinite number of nonprincipal primes outside the set $\left\{P_{\alpha}\right\}$.

CoRollary 1-8. Let $A$ be a Dedekind domain with at least one prime ideal in every ideal class. Then for any multiplicatively closed set $S, A_{S}$ will have a prime ideal in every class except possibly the principal class.

Proof. By Corollary 1-3, every class of $A_{S}$ is the image of a class of $A$. Let $\bar{D}$ be a non-principal class of $A_{S} . \quad \bar{D}=\overline{C A}_{S}$, where $C$ is a fractionary ideal of $A$. By assumption, there is a prime $P$ of $A$ such that $\bar{P}=\bar{C}$. If $P A_{S}=A_{s}$, then $C A_{S}$ is principal and so $\bar{D}$ is the principal class of $A_{S}$. This is not the case, so $P A_{S}$ is prime, and certainly $\overline{P A}_{S}=\overline{C A}_{S}=\bar{D}$.

EXAMPLE 1-9. There is a Dedekind domain which is not the integral closure of a principal ideal domain.

Let $A=Z[\sqrt{-5}] . \quad A$ is a Dedekind domain which is not a principal ideal domain. In $A, 29=(3+2 \sqrt{-5}) \cdot(3-2 \sqrt{-5})$. It follows from elementary algebraic number theory that $I_{1}=3+2 \sqrt{-5}$ and $\Pi_{2}=3-2 \sqrt{-5}$ generate distinct prime ideals of $A$. Let $S=$ $\left\{\Pi_{1}^{k}\right\}_{k \geqq 0}$. Then $A_{S}$ is by Theorem $1-4$ a Dedekind domain which is not a principal ideal domain. Let $F$ denote the quotient field of $A$ and $Q$ the rational numbers. $A_{S}$ cannot be the integral closure of a principal ideal domain whose quotient field is $F$ since principal ideal domains are integrally closed. If $A_{S}$ were the integral closure of a principal ideal domain $C$ with quotient field $Q$, then $C$ would contain $Z$, and $\Pi_{1}$ and $\Pi_{2}$ would be both units or nonunits in $A_{S}$ (since $\Pi_{1}$ and $\Pi_{2}$ are conjugate over $Q$ ). But only $\Pi_{1}$ is a unit in $A_{S}$.

REMARK 1-10. Example 1-9 settles negatively a conjecture in Vol. I of Commutative Algebra [2, p. 284]. The following conjecture may yet be true: Every Dedekind domain can be realized as an $A_{s}$, where $A$ is the integral closure of a principal ideal domain in a finite extension field and $S$ is a multiplicatively closed set of $A$. 
2. Lemma 2-1. Let $A$ be a Dedekind domain. Let $S$ be the multiplicatively closed set of $A[X]$ consisting of all monic polynomials of $A[X]$. Let $T$ be the multiplicatively closed set of all primitive polynomials of $A[X]$ (i.e. all polynomials whose coefficients generate the unit ideal of $A$ ). Then $A[X]_{S}$ and $A[X]_{T}$ are both Dedekind domains.

Proof. $A[X]$ is integrally closed and noetherian, and so both $A[X]_{S}$ and $A[X]_{T}$ are integrally closed and noetherian. Let $P$ be a prime ideal of $A[X]$. If $P \cap A \neq(0)$, then $P \cap A=Q$ is a maximal ideal of $A$. If $P \neq Q A[X]$, then passing to $A[X] / Q A[X]$, it is easy to see that $P=Q A[X]+f(X) \cdot A[X]$ where $f(X)$ is a suitably chosen monic polynomial of $A[X]$. In this case $P \cap S \neq \phi$, so $P A[X]_{S}=A[X]_{S}$. Thus if $P \cap A \neq(0)$ and $\mathrm{P} A[X]_{S}$ is a proper prime of $A[X]_{S}$, then $P=Q A[X]$ where $Q=P \cap A$. Then height $P=$ height $Q=1$. If $P \cap A=(0)$, then $P K[X]$ is a prime ideal of $K[X]$ (where $K$ denotes the quotient field of $A$ ). Certainly height $P=$ height $P K[X]=1$, so in any case if a prime $P$ of $A[X]$ is such that $P \cap S=\phi$, then height $P \leqq 1$. This proves that $A[X]_{S}$ is a Dedekind domain. Since $S \subseteq T$, $A[X]_{T}$ is also a Dedekind domain by Lemma $1-1$.

REMARK 2-2. $A[X]_{T}$ is customarily denoted by $A(X)[1$, p. 18]. For the remainder of this article, $A[X]_{S}$ will be denoted by $A^{1}$.

Proposition 2-3. $A^{1}$ has the same ideal class group as $A$. In fact, the map $\bar{C} \rightarrow \overline{C^{\prime} A^{1}}$ is a one-to-one map of the ideal class group of $A$ onto that of $A^{1}$.

We can prove that $\bar{C} \rightarrow \overline{C A^{1}}$ is a one-to-one map of the ideal class of $A$ into that of $A$ by showing that if two integral ideals $D$ and $E$ of $A$ are not in the same class, neither are $D A^{1}$ and $E A^{1}$. Suppose then that $\overline{D A^{1}}=\overline{E A^{1}}$. This implies that there are elements $f_{i}(X)$, $g_{i}(X), i=1,2$ in $A[X]$ with $g_{i}(X)$ monic for $i=1,2$ such that

$$
D A^{1} \cdot \frac{f_{1}(X)}{g_{1}(X)}=E A^{1} \cdot \frac{f_{2}(X)}{g_{2}(X)} .
$$

Let $a_{i}$ be the leading coefficient of $f_{i}(X)$ for $i=1,2$, and let $d \in D$. Then we get a relation

$$
d \cdot \frac{f_{1}(X)}{g_{1}(X)}=\frac{e(X)}{g(X)} \cdot \frac{f_{2}(X)}{g_{2}(X)}, g(X) \text { monic, }
$$

where $e(X)$ can be chosen as a polynomial in $A[X]$ all of whose coefficients are in $E$. This leads to $d g_{2}(X) \cdot f_{1}(X) \cdot g(X)=e(X) \cdot f_{2}(X) \cdot g_{1}(X)$. The leading coefficient on the right is in $a_{2} \cdot E$. This shows that $a_{1} \cdot D$ 
$D \leqq a_{2} \cdot E$. Likewise $a_{2} \cdot E \cong a_{1} \cdot D$, thus $a_{1} \cdot D=a_{2} \cdot E$ and $\bar{D}=\bar{E}$.

To prove the map is onto, the following lemma is needed.

Lemma 2-4. Let $A$ be a Dedekind domain with quotient field $K$. To each polynomial $f(X)=a_{n} X^{n}+\cdots-+a_{o}$ of $K[X]$ assign the fractionary ideal $c(f)=\left(a_{n}, \cdots, a_{o}\right)$. Then $c(f g)=c(f) c(g)$.

Proof. Let $V_{p}$ (for each prime $P$ of $A$ ) denote the $P$-adic valuation of $A$. It is immediate that $V_{p}(c(f))=\min V_{p}\left(a_{i}\right)$. Because of the unique factorization of fractionary ideals in Dedekind domains, it suffices to show that $V_{p}(c(f g))=V_{p}(c(f))+V_{p}(c(g))$ for each prime $P$ of $A$. This will be true if the equation is true in each $A_{p}[X]$. But $A_{p}$ is a principal ideal domain, and the well-known proof for principal ideal domains shows the truth of the lemma.

To complete Prop. 2-3, let $P$ be a prime ideal of $A^{1}$. The proof of Lemma 2-1 shows that if $P \cap A \neq(0)$, then $P=Q A^{1}$ where $Q$ is a prime of $A$. Thus $\bar{P}=\overline{Q A^{1}}$ and ideal classes generated by these primes are images of classes of $A$. Suppose now that $P$ is a prime of $A^{1}$ such that $P \cap A=(0)$. Let $P^{1}=P \cap A[X]$. Then $P^{1} \cap A=(0)$, and $P^{1} \cdot K[X]$ is a prime ideal of $K[X]$. Let $P^{1} \cdot K[X]=f(X) K[X]$; we may choose $f(X)$ in $A[X]$. Let $C=c(f)$. Suppose that $g(X) \cdot f(X) \in$ $A[X]$. Then because $c(f g)=(c(f))+(c(g)) \geqq 0$ for all $P, g(X) \in C^{-1}$. $A[X]$. Conversely if $g(X) \in C^{-1} \cdot A[X]$, then $g(X) f(X) \in A[X]$. Thus $P^{1}=f(X) K[X] \cap A[X]=C^{-1} \cdot A[X] \cdot f(X) A[X]$, and $P=P^{1} A^{1}=C^{-1}$. $A^{1} \cdot f(X) A^{1}$. This gives finally that $\bar{P}=\overline{C^{-1} A^{1}}$, and the class is an image of a class of $A$ under our map. Since the ideal class group of $A^{1}$ is generated by all $\bar{P}$ where $P$ is a prime of $A^{1}$, this finishes the proof.

Corollary 2-5. A1 has a prime ideal in each ideal class.

Proof. Let $w$ be any nonunit of $A$. Then $(w X+1) K[X] \cap A^{1}$ $\left(=(w X+1) A^{1}\right)$ is a prime ideal in the principal class. Otherwise let $C$ be any integral ideal in a nonprincipal class $\bar{D}^{-1} \cdot C$ can be generated by 2 elements, so suppose $C=\left(c_{0}, c_{1}\right)$; then $Q=\left(c_{0}+c_{1} X\right) \cdot$ $K[X] \cap A^{1}$ is a prime ideal in $\overline{C^{-1} A^{1}}=\bar{D}$.

Proposition 2-6. If $A$ is a Dedekind domain, then $A(X)$ is a principal ideal domain.

Proof. Since $A(X)=A_{T}^{1}$, Corollary 1-3 and the proof of Corollary 2-5 show that each nonprincipal class of $A(X)$ contains a prime $Q A(X)$, where $Q$ is a prime ideal of $A$ of the type $\left(c_{0}+c_{1} X\right) K[X] \cap A^{1}$. Clearly $Q \cap A[X]=\left(c_{0}+c_{1} X\right) K[X] \cap A[X]=C^{-1} \cdot A[X] \cdot\left(c_{0}+c_{1} X\right) A[X] \nsubseteq$ 
$P A[X]$ for any prime $P$ of $A$. Thus there is in $Q \cap A[X]$ a primitive polynomial of $A[X \mid$. Thus $Q A(X)=A(X)$. Theorem 1-4 now implies that every class of $A$ becomes principal in $A(X)$, i.e. $A(X)$ is a principal ideal domain.

REMARK 2-7. Proposition 2-6 is interesting in light of the fact that the primes of $A(X)$ are exactly those of the form $P A(X)$, where $P$ is a prime of $A[1, \mathrm{p} .18]$.

REMARK 2-8. If the conjecture given in Remark 1-10 is true for a Dedekind domain $A$, it is also true for $A^{1}$. For suppose $A=B_{a r}$, where $M$ is a multiplicatively closed set of $B$ and $B$ is the integral closure of a principal ideal domain $B_{0}$ in a suitable finite extension field. Let $S, S^{1}$, and $T$ be the set of monic polynomials in $A[X]$, $B[X]$, and $B_{0}[X]$ respectively. Then $A^{1}=A[X]_{S}=\left(B_{M}[X]\right)_{S}=$ $\left(B[X]_{M}\right)_{S}=(B[X])_{\langle M, S\rangle}=\left(B[X]_{S^{1}}\right)_{\langle M, S\rangle}$. The last equality holds because $S^{1} \subseteq S \subseteq\langle M, S\rangle$. It is easy to see that $B[X]_{S^{1}}$ is the integral closure of the principal ideal domain $B_{0}[X]_{T}$ in $K(X)$, where $K$ is the quotient field of $B$.

\section{REFERENCES}

1. M. Nagata, Local rings, New York, Interscience Publishers, Inc. (1962).

2. O. Zariski and P. Samuel, Commutative algebra, Vol. I, Princeton, D. Van Nostrand Company (1958).

Cornell College 


\section{PACIFIC JOURNAL OF MATHEMATICS}

\section{EDITORS}

H. Samelson

Stanford University

Stanford, California

R. M. Blumenthal

University of Washington

Seattle, Washington 98105
J. Dugundu

University of Southern California Los Angeles, California 90007

*Richard Arens

University of California

Los Angeles, California 90024

\section{ASSOCIATE EDITORS}
E. F. BECKENBACH
B. H. NeumanN
F. WOLF
K. YOSIDA

\section{SUPPORTING INSTITUTIONS}

UNIVERSITY OF BRITISH COLUMBIA CALIFORNIA INSTITUTE OF TECHNOLOGY UNIVERSITY OF CALIFORNIA MONTANA STATE UNIVERSITY

UNIVERSITY OF NEVADA

NEW MEXICO STATE UNIVERSITY

OREGON STATE UNIVERSITY

UNIVERSITY OF OREGON

OSAKA UNIVERSITY

UNIVERSITY OF SOUTHERN CALIFORNIA
STANFORD UNIVERSITY

UNIVERSITY OF TOKYO

UNIVERSITY OF UTAH

WASHINGTON STATE UNIVERSITY

UNIVERSITY OF WASHINGTON

AMERICAN MATHEMATICAL SOCIETY CALIFORNIA RESEARCH CORPORATION SPACE TECHNOLOGY LABORATORIES NAVAL ORDNANCE TEST STATION 


\section{Pacific Journal of Mathematics}

\section{Vol. 15, No. $1 \quad$ September, 1965}

Donald Charles Benson, Unimodular solutions of infinite systems of linear

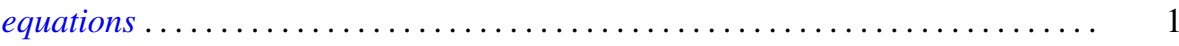

Richard Earl Block, Transitive groups of collineations on certain designs . . . . . . 13

Barry William Boehm, Existence of best rational Tchebycheff approximations .... . 19

Joseph Patrick Brannen, A note on Hausdorff's summation methods . . . . . . . . . . 29

Dennison Robert Brown, Topological semilattices on the two-cell ............ 35

Peter Southcott Bullen, Some inequalities for symmetric means . . . . . . . . . . 47

David Geoffrey Cantor, On arithmetic properties of coefficients of rational

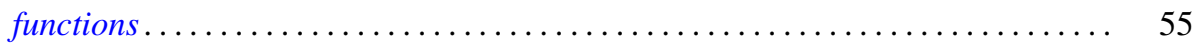

Luther Elic Claborn, Dedekind domains and rings of quotients . . . . . . . . . 59

Allan Clark, Homotopy commutativity and the Moore spectral sequence ........ 65

Allen Devinatz, The asymptotic nature of the solutions of certain linear systems of

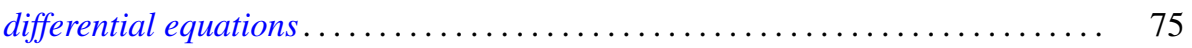

Robert E. Edwards, Approximation by convolutions ................... 85

Theodore William Gamelin, Decomposition theorems for Fredholm operators . . . . . 97

Edmond E. Granirer, On the invariant mean on topological semigroups and on

topological groups .................................. 107

Noel Justin Hicks, Closed vector fields . . . . . . . . . . . . . . . . . . . 141

Charles Ray Hobby and Ronald Pyke, Doubly stochastic operators obtained from

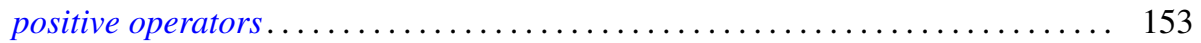

Robert Franklin Jolly, Concerning periodic subadditive functions . . . . . . . . . 159

Tosio Kato, Wave operators and unitary equivalence . . . . . . . . . . . . . . 171

Paul Katz and Ernst Gabor Straus, Infinite sums in algebraic structures . . . . . . . 181

Herbert Frederick Kreimer, Jr., On an extension of the Picard-Vessiot theory ...... 191

Radha Govinda Laha and Eugene Lukacs, On a linear form whose distribution is

identical with that of a monomial ......................... 207

Donald A. Ludwig, Singularities of superpositions of distributions . . . . . . . . . 215

Albert W. Marshall and Ingram Olkin, Norms and inequalities for condition

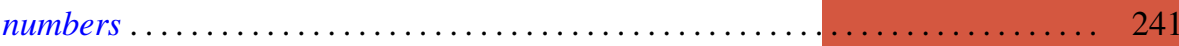

Horace Yomishi Mochizuki, Finitistic global dimension for rings . . . . . . . . . . 249

Robert Harvey Oehmke and Reuben Sandler, The collineation groups of division

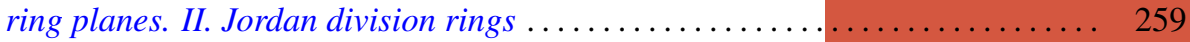

George H. Orland, On non-convex polyhedral surfaces in $E^{3} \ldots \ldots \ldots \ldots \ldots \ldots \ldots 267$

Theodore G. Ostrom, Collineation groups of semi-translation planes . . . . . . . . 273

Arthur Argyle Sagle, On anti-commutative algebras and general Lie triple

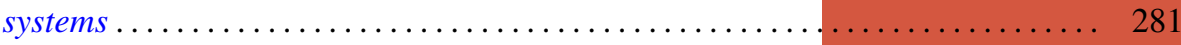

Laurent Siebenmann, A characterization of free projective planes . . . . . . . . . 293

Edward Silverman, Simple areas.................................. 299

James McLean Sloss, Chebyshev approximation to zero .................. 305

Robert S. Strichartz, Isometric isomorphisms of measure algebras . . . . . . . . . 315

Richard Joseph Turyn, Character sums and difference sets . . . . . . . . . . . . 319

L. E. Ward, Concerning Koch's theorem on the existence of arcs . . . . . . . . . . 347

Israel Zuckerman, A new measure of a partial differential field extension ......... 357 